\title{
Social distance capacity to control the COVID-19 pandemic: A systematic review on time series analysis
}

\author{
Omid Khosravizadeh $^{\mathrm{a}}$, Bahman Ahadinezhad ${ }^{\mathrm{a}}$, Aisa Maleki ${ }^{\mathrm{b}, *}$, Zahra Najafpour ${ }^{\mathrm{c}}$ and \\ Rohollah Golmohammadic \\ ${ }^{a}$ Social Determinants of Health Research Center, Research Institute for Prevention of \\ Non-Communicable Diseases, Qazvin University of Medical Sciences, Qazvin, Iran \\ ORCIDs: https://orcid.org/0000-0001-6893-3489 (OK); https://orcid.org/0000-0001-6940-3498 (BA) \\ ${ }^{\mathrm{b}}$ Health Products Safety Research Center, Qazvin University of Medical Sciences, Qazvin, Iran \\ ORCID: https://orcid.org/0000-0001-5706-0993 (AM) \\ ${ }^{\mathrm{c}}$ Student Research Committee, Qazvin University of Medical Sciences, Qazvin, Iran \\ ORCIDs: https://orcid.org/0000-0001-5577-0975 (ZN); https://orcid.org/0000-0001-7359-2236 (RG)
}

Received 27 June 2021

Accepted 10 October 2021

\begin{abstract}
.
BACKGROUND: Reducing interpersonal contact has been one of the least expensive and most widely used COVID-19 control strategies.

OBJECTIVE: This systematic review has been conducted with the aim of identifying social distancing strategies and policies and their impact on the COVID-19 pandemic.

METHODS: In order to compile this systematic review, Google Scholar, PubMed, Scopus, Web of Science, Science Direct, Magiran, SID, and Irandoc databases were searched from the COVID-19 outbreak until March 2021. Keywords included "social", "physical", "distance", "outbreak", "incidence", "prevalence", "spread", "new case", "death*”, "mortality*”, "morbidity*”, "covid-19", "coronavirus", "sars-cov-2" and "time series*". The articles were qualitatively evaluated by two researchers using the STROBE tool. Finally, the study data were divided into three conceptual categories by three researchers, who then agreed on one category. The practical suggestions were also categorized in the same way.

RESULTS: The policies and strategies adopted to implement social distancing were included in five categories of restrictions, prohibitions, closures, incentives, and punishments. Transportation and travel restrictions, crowded places and schools closure, use of telecommunications and virtual communications, and financial and psychological support to society members were the main policies in this area.

CONCLUSION: Rapid and complete vaccination of all people around the world is out of reach, therefore social distancing and the implementation of physical restraints, especially in crowded and densely populated environments, should be done extensively until COVID-19 is eradicated.
\end{abstract}

Keywords: Social distance, new cases, death, COVID-19, coronavirus, time series, health

\footnotetext{
*Address for correspondence: Aisa Maleki, Health Products Safety Research Center, Qazvin University of Medical Sciences, Qazvin, Iran. Tel.: +98 9223695917; E-mail: aisamalekii@gmail.com.
} 


\section{Introduction}

Acute respiratory syndrome is an infectious disease caused by a new type of coronavirus known as COVID-19, which broke out in Wuhan in December 2019 [1-3]. COVID-19 is transmitted from human to human through respiratory droplets, cough, sneezing, and Surface contamination and has a high transmission rate; Asymptomatic patients can also be a source of infection [4,5]. The devastating effects of this pandemic on various aspects of the lives of societies, both economic and social, led the World Health Organization (WHO) to declare this crisis a Public Health Emergency of International Concern [6]. According to the WHO Situation Report, on 13 February 2021, 107,838,255 COVID-19 cases were confirmed and 2,373,398 COVID-19 deaths occurred worldwide. According to these statistics, COVID-19 confirmed cases and deaths are 48,021,725 and 1,127,620 in the United States, 36,436,128 and 809,441 in Europe, 13,165,612 and 202,222 in Southeast Asia, 5,976,060 and 139,129 in Africa [7]. The incubation period of this disease is 1-14 days and on average 5-6 days after exposure to the infected person, symptoms such as dry cough, fever, anorexia, body fatigue, and loss of smell and taste occur $[3,8,9]$. In the early strains of the virus, on average, people over the age of 60 and those with at least one underlying disease such as cardiovascular disease, hypertension, and diabetes were at higher risk for severe complications, approximately $65 \%$ of patients were male and $35 \%$ were female, and the mortality rate increased over the age of 80 years [2,3]. However, in the new mutations of COVID-19, the rate of infection and risk of serious complications and death is higher in all age groups, especially in youth and children [10]. In the early stages of the COVID-19 pandemic, due to the lack of effective medications and vaccines, many countries considered prevention to be the most effective way to control and inhibit COVID-19 [1]. Currently, the WHO has taken action for the equitable distribution and vaccine access for all people in the world, regardless of their country's economic situation, through Kovacs due to the limited number of vaccines [11]. Since vaccine resources are limited and global vaccination is time-consuming, the importance of observing restrictions such as social distance alongside vaccination is still emphasized [12]. In this regard, the WHO introduced wearing a mask, quarantine, social distance, and 20-second hand-washing $[8,13]$. Many countries have adopted a policy of physical distancing to overcome this pandemic and mitigate its consequences [4]. Social distancing, continuous disinfecting, air conditioning, staying home, and hand washing are some of the policies that have reduced the prevalence of COVID-19 in South Korea [14]. Wearing masks in public places, canceling gatherings, controlling body temperature, maintaining social distance were enforced in Hangzhou, China to curb the pandemic, and every household had the right to leave home every two days to buy necessities, which ultimately reduced the prevalence of COVID-19 [15]. The United States also implemented a strategy to limit physical and social contact between individuals. The policies of various states included restricting restaurants, closing schools, ordering people to stay home, issuing emergency notices, canceling gatherings, and closing unnecessary businesses. These policies reduced the individual's movement by $1-5 \%$ in the first five days and by $4.5-7 \%$ after 20 days [16]. A study in South Korea found that the prevalence of COVID-19 in workplaces with high population density was very high and these places could be a strong source of virus transmission, so the social distancing strategy in the workplace with appropriate guidelines is one of the most important ways to reduce the prevalence of COVID-19 [17]. A study in the United States found that public policies were able to reduce the average number of COVID-19 new cases by $0.08 \%$; Therefore, in order to gain support for the social distancing policy, one must pay attention to public policies [18]. Non-pharmacological solutions, along with widespread global vaccination, are effective 
ways to curb COVID-19 and its complications. In this regard, social distancing strategies and policies are some of the most popular strategies adopted by various governments. It is also possible to apply different levels of this policy in most cities and societies with different structures. On the other hand, this policy overshadows the mental health of society's individuals by isolating them. It indicates the need to examine the effectiveness of this policy to notice whether it is worth trading off with its consequences. Therefore, this systematic review aims to identify the social distancing strategies and policies adopted around the world and to examine their impact on the COVID-19 pandemic through time-series studies.

\section{Materials and methods}

\subsection{Study design}

This systematic review is based on a comprehensive examination of the Google Scholar, PubMed, Scopus, Web of Science, Science Direct, Magiran, SID, and Irandoc databases from the COVID19 outbreak until March 2021. Preferred Reporting Items for Systematic Reviews and Meta-Analyses (PRISMA) was used as a literature review guide [19].

\subsection{Research questions}

In this review, the following questions were answered:

Question (1) What are the social distancing policies and strategies and what are the practical suggestions for its effective implementation?

Question (2) What effect has the social distancing policy had on the prevalence, incidence, spread, and new cases of COVID-19?

\subsection{Search strategy}

The following keywords were searched: "social", "physical", "distance", "outbreak", "incidence", "prevalence", "spread", "new case", "death*”, "mortality*”, "morbidity*”, "covid-19", "coronavirus", "sars-cov-2" and "time series*". Some key journals were manually searched to identify all related articles. The search period was confined to the COVID-19 outbreak (2019 to March 2021) (Table 1).

\subsection{Inclusion and exclusion criteria}

Research articles, reviews, empirical researches, and book chapters on the social distancing policy during the COVID-19 outbreak, publications that examined the outbreak, incidence, prevalence, spread, and new cases of COVID-19 in Persian and English were included in this study. Meanwhile, secondary data, conference abstracts and editorials were removed. Studies on the effect of social distancing on other diseases were also not reviewed. In addition, studies on social distancing during the COVID-19 pandemic were screened with two major exclusion criteria: (1) Studies performed using non-time series methods, and (2) Studies that did not report desired indicators values before and after the intervention. The logic of such screening was that time-series studies include a set of statistical data that is collected at regular intervals. It is a widely used and valid method in analyzing patterns over time and analyzing interventions. It also reports the quantitative and objective impact of the intervention at several time points and its effectiveness trend [20]. 
Table 1

Search strategy

\begin{tabular}{|c|c|}
\hline Databases & Search strategy \\
\hline Web of Science & $\begin{array}{l}(((\mathrm{TI}=\text { social OR TI = physical }) \text { AND TI }=\text { distance AND }(\mathrm{TI}=\text { Outbreak OR TI = "new case" } \\
\text { OR TI = Incidence OR TI = Prevalence OR TI = spread OR TI = death* OR TI = mortality* OR } \\
\text { TI = morbidity*) OR ((AB = social OR AB = physical }) \text { AND AB = distance AND (AB = } \\
\text { Outbreak OR AB = "new case" OR AB = Incidence OR AB = Prevalence OR AB = spread OR } \\
\text { AB = death* OR AB = mortality* OR AB = morbidity* })) \text { AND (AB = Covid-19 OR AB = } \\
\text { Sars-cov-2 OR AB = Coronavirus }) \text { AND AB = "time series*”) AND LANGUAGE:(English) } \\
\text { ANDDOCUMENT TYPES:(Article) } \\
\text { Indexes = SCI-EXPANDED, SSCI, A\&HCI, CPCI-S, CPCI-SSH, BKCI-S, BKCI-SSH, ESCI, } \\
\text { CCR-EXPANDED, IC Timespan = 2019-2021 }\end{array}$ \\
\hline PubMed & $\begin{array}{l}(((((\text { social[Title/Abstract]) OR (physical[Title/Abstract]]) AND (distance[Title/Abstract])) AND } \\
((((((\text { outbreak[Title/Abstract]) OR (death*[Title/Abstract])) OR (incidence[Title/Abstract])) OR } \\
\text { (prevalence[Title/Abstract])) OR (“new case”[Title/Abstract])) OR (mortality*[Title/Abstract])) } \\
\text { OR (morbidity*[Title/Abstract]))) AND (((Covid-19[Title/Abstract]) OR } \\
\text { (Sars-cov-2[Title/Abstract])) OR (Coronavirus[Title/Abstract]))) AND (“time } \\
\text { series"[Title/Abstract]) AND (2019:2021[pdat])) Filters: from 2019-2021 }\end{array}$ \\
\hline Scopus & $\begin{array}{l}\text { (TITLE-ABS-KEY (social OR physical) AND TITLE-ABS-KEY (distance)) AND } \\
\text { (TITLE-ABS-KEY (Covid-19 OR Sars-cov-2 OR Coronavirus) AND TITLE-ABS-KEY } \\
\text { (outbreak OR incidence OR prevalence OR spread OR “new case" OR death* OR mortality* OR } \\
\text { morbidity*) AND TITLE-ABS-KEY (“time series*”)) AND PUBYEAR > } 2018 \text { AND } \\
\text { DOCTYPE (ar) AND PUBYEAR > } 2018\end{array}$ \\
\hline Google Scholar & 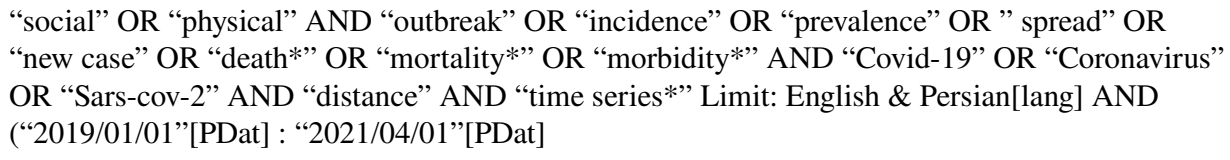 \\
\hline Science Direct & $\begin{array}{l}\text { Title, abstract, keywords: "social" OR "physical” AND “outbreak" OR "incidence" OR } \\
\text { "prevalence" OR " spread" OR "new case" OR "death*” OR “mortality*” OR “morbidity*” AND } \\
\text { "Covid-19" OR "Coronavirus" OR "Sars-cov-2" AND "distance" AND "time series*”, limited to: } \\
2019-2021\end{array}$ \\
\hline Other & 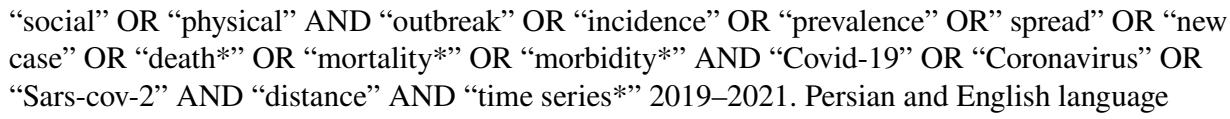 \\
\hline
\end{tabular}

\subsection{Review process}

First, the articles were entered into resource management software (EndNote X8) and duplicates were removed. In the next step, two researchers reviewed and screened the abstracts. Articles that answered the research questions were retained and their resources were added for further coverage. These articles were matched with inclusion and exclusion criteria and eligible articles were inclusion in the study.

\subsection{Quality assessment}

After reviewing the articles, three researchers conducted a qualitative assessment of the remaining studies. The STROBE checklist was used to assess the quality of observational studies. STROBE contains 


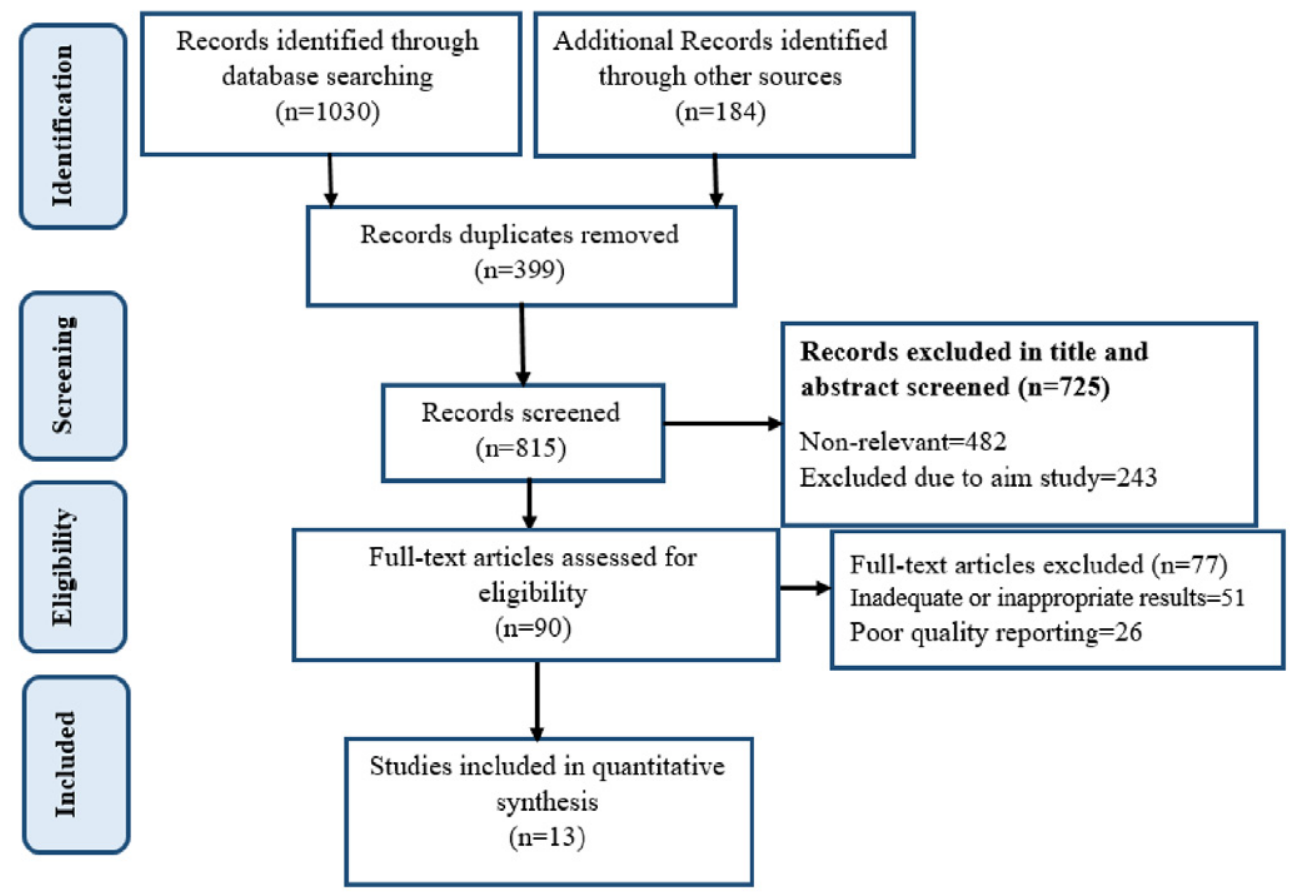

Fig. 1. Flow diagram of the literature search.

22 components that cover different parts of the article [21]. Articles that showed more than $50 \%$ compliance with the checklist were included in the study.

\subsection{Data extraction}

After assessing the quality of the articles, 13 were selected and their related data including author/year of publication, study setting, study design, method, and a concise report of key findings were extracted in accordance with PRISMA provisions. Finally, the study data were divided into three conceptual categories by three researchers, and the researchers agreed on one category. The practical suggestions were also categorized in the same way.

\subsection{Ethical approval}

Ethical approval was obtained from the ethics committee of Qazvin University of Medical Sciences (ethics code IR. QUMS. REC. 1400. 201).

\section{Results}

1214 publication were found, which included 399 duplicates and were thus omitted. 725 studies was also rejected after reviewing the titles and abstracts. A full text review furthermore excluded 77 articles. Finally, 13 articles were included in the study to answer the research question (Fig. 1). 
Data related to the 13 articles were reviewed, including author name, publication year, statistical population, study purpose, type of study, and a summary of key findings (Table 2). A review of time series studies on social distancing has shown that this strategy has been effective in China, most European countries including Sweden, Norway, Italy and Spain, as well as the United States, Brazil, Australia, New Zealand and Iran. In Indonesia, social distance had no statistically significant effect on the COVID-19 pandemic.

A review of studies showed that the policies and strategies adopted to implement social distancing were included in five categories of restrictions. (1) Policies that control and restrict contact between people, prohibitions; (2) Policies that prohibit and cut off contact between people, closures; (3) Policies that completely prevent people from attending in places and contacting each other by closing places, incentives; (4) Policies that involve individuals in the implementation of preventive measures, through culture-building and economic supports, and punishments; (5) Policies that use coercion to force people to abide by protocols (Table 3 ).

The most effective measures used in the studies under review, along with the authors' points of view, are summarized in the form of practical suggestions for the effective implementation of social distancing in accordance with the above categories and are presented in Table 4.

\section{Discussion}

Numerous measures have been taken to reduce the burden of COVID-19 and its side effects, ranging from quarantine to wearing a mask. Due to the high prevalence of coronavirus, the severe burden of this disease on health systems, and the lack of financial and human resources, effective strategies should be adopted to reduce the prevalence of COVID-19 infection. One of the strategies that have been implemented in many countries so far is social distancing, which means reducing the contact rate of individuals. The present study, in the form of a systematic review, seeks to answer the question of whether social distance affects the prevalence of COVID-19 and what are its policies and strategies? In response to this question, it was found that social distance in the country where this new virus originated, China, as well as many other countries including the United States, Norway, Sweden, Brazil, Iran, Italy, Spain, Australia, and New Zealand has been effective. Therefore, in these countries, after the implementation of this strategy, a significant reduction in the prevalence of COVID-19 was observed.

Given that it takes time to complete vaccinations globally, especially in developing countries, the best approach now is to follow social distance policies along with extensive testing [37]. Many other studies agree with this finding; Thailand, China, New Zealand, and Hong Kong have introduced social distancing as an effective policy [38-40].

Policies and strategies for enforcing social distancing fall into five general categories: restrictions, prohibitions, closures, incentive, and punishment policies. In line with the closure policy, many studies stated that school closures were associated with a significant reduction in the prevalence of airborne infectious diseases incidence and mortality such as colds, gastroenteritis, bronchiolitis, and coronavirus [41,42]. Since population movements in public places are a major cause of human contact and transmission of the virus, the closure of these places could be one of the first measures that can be imposed. Closure of places and communities has different effects in different contexts. In developing countries, which often do not have a strong infrastructure for telecommunications and online classes and meetings, educational and work productivity declines. On the other hand, working at home while reducing social interactions may upset the balance of work and life.

Regarding restrictive policies, a study by Atalan et al. also highlighted that preventive social restriction could significantly reduce the prevalence of the virus. One of the policies they have introduced in this 
O. Khosravizadeh et al. / Social distance capacity to control the COVID-19 pandemic

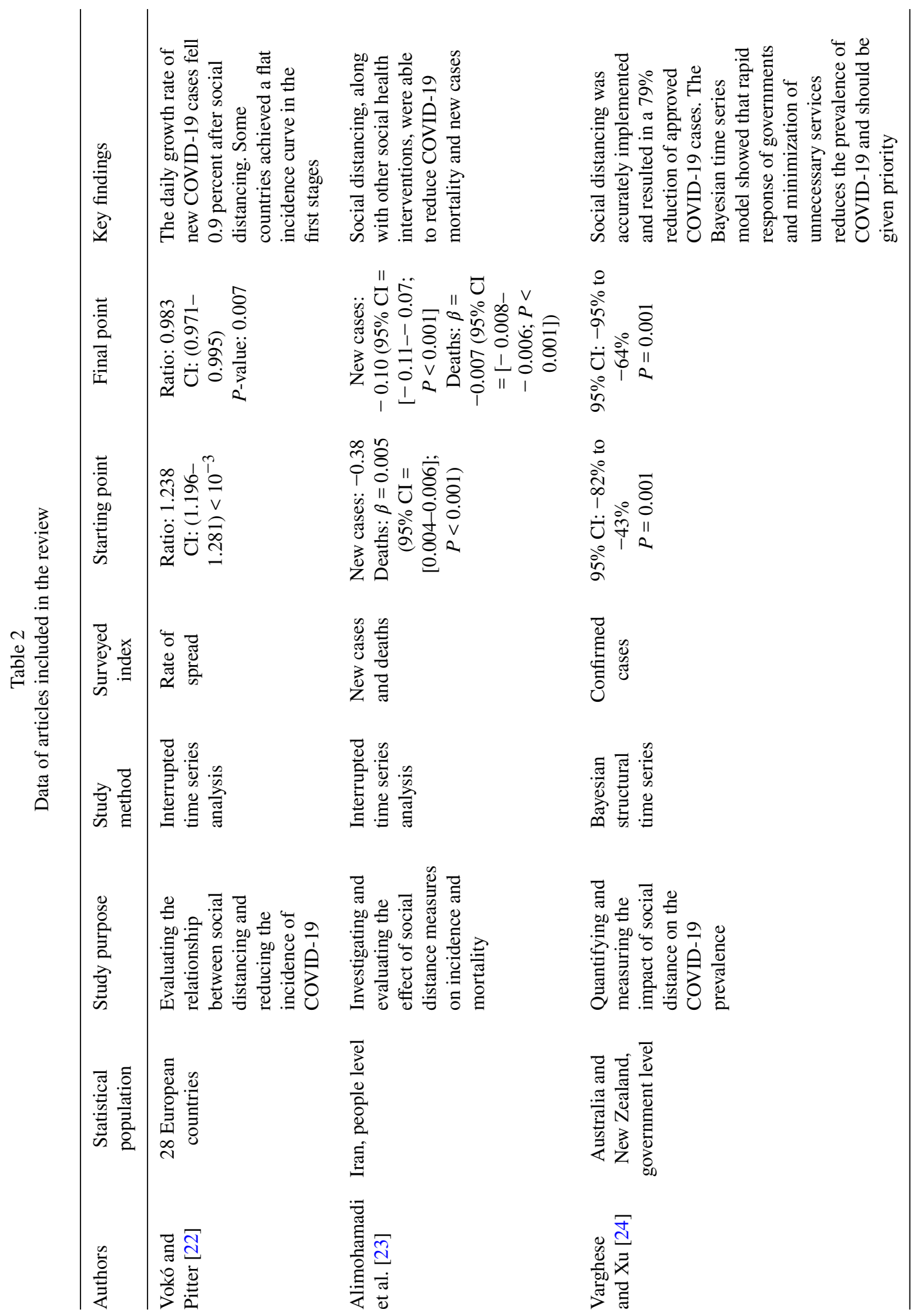




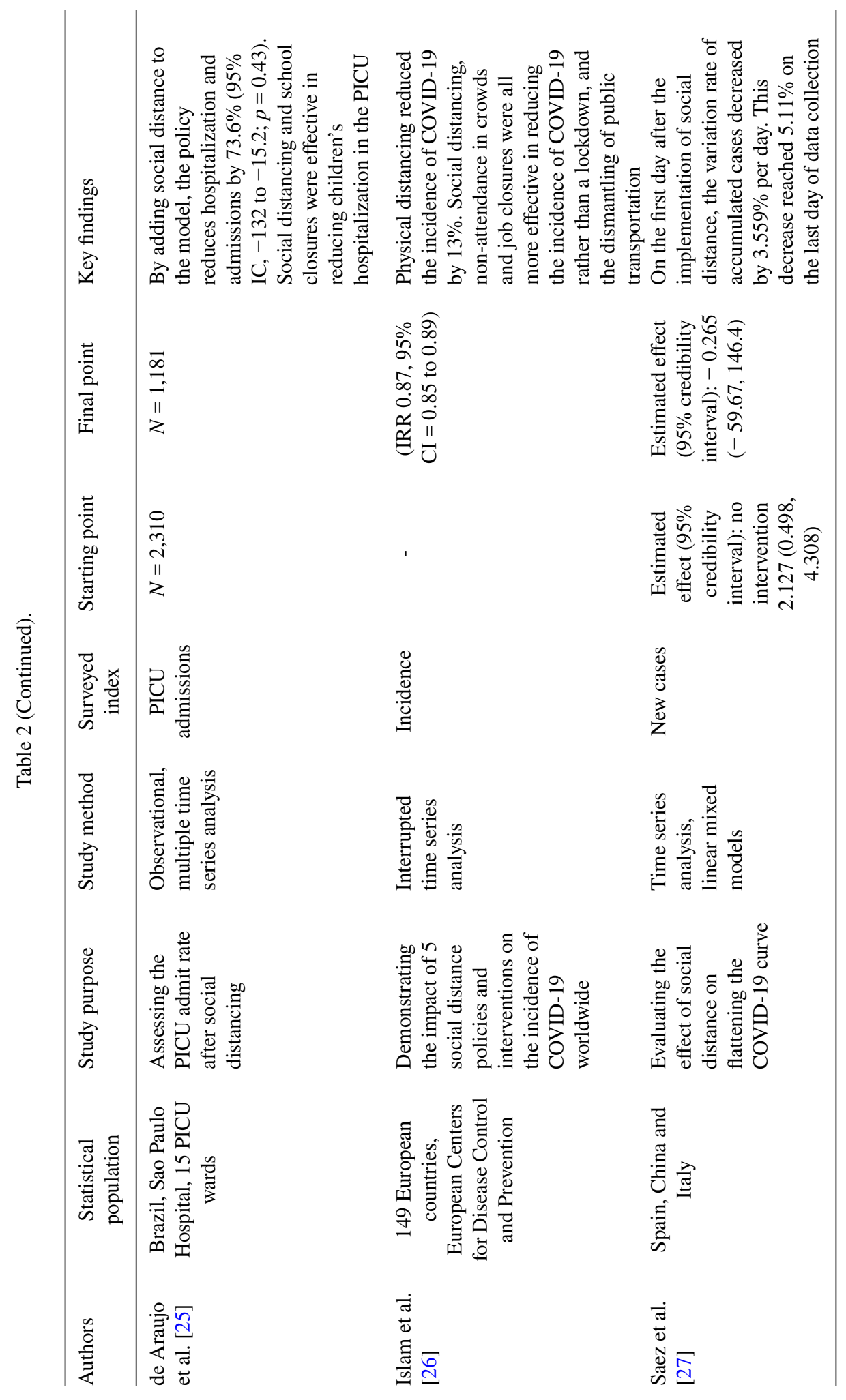




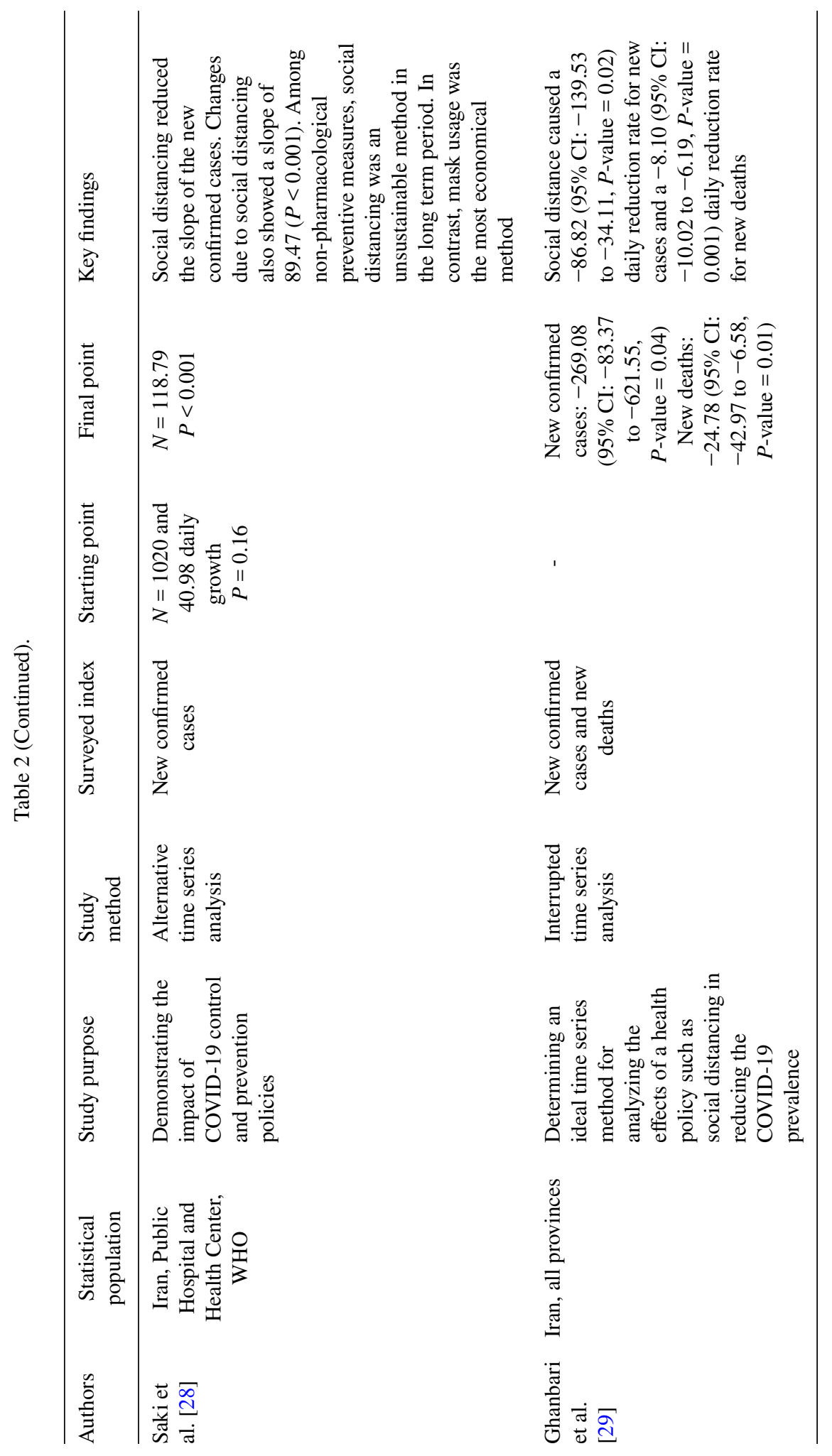




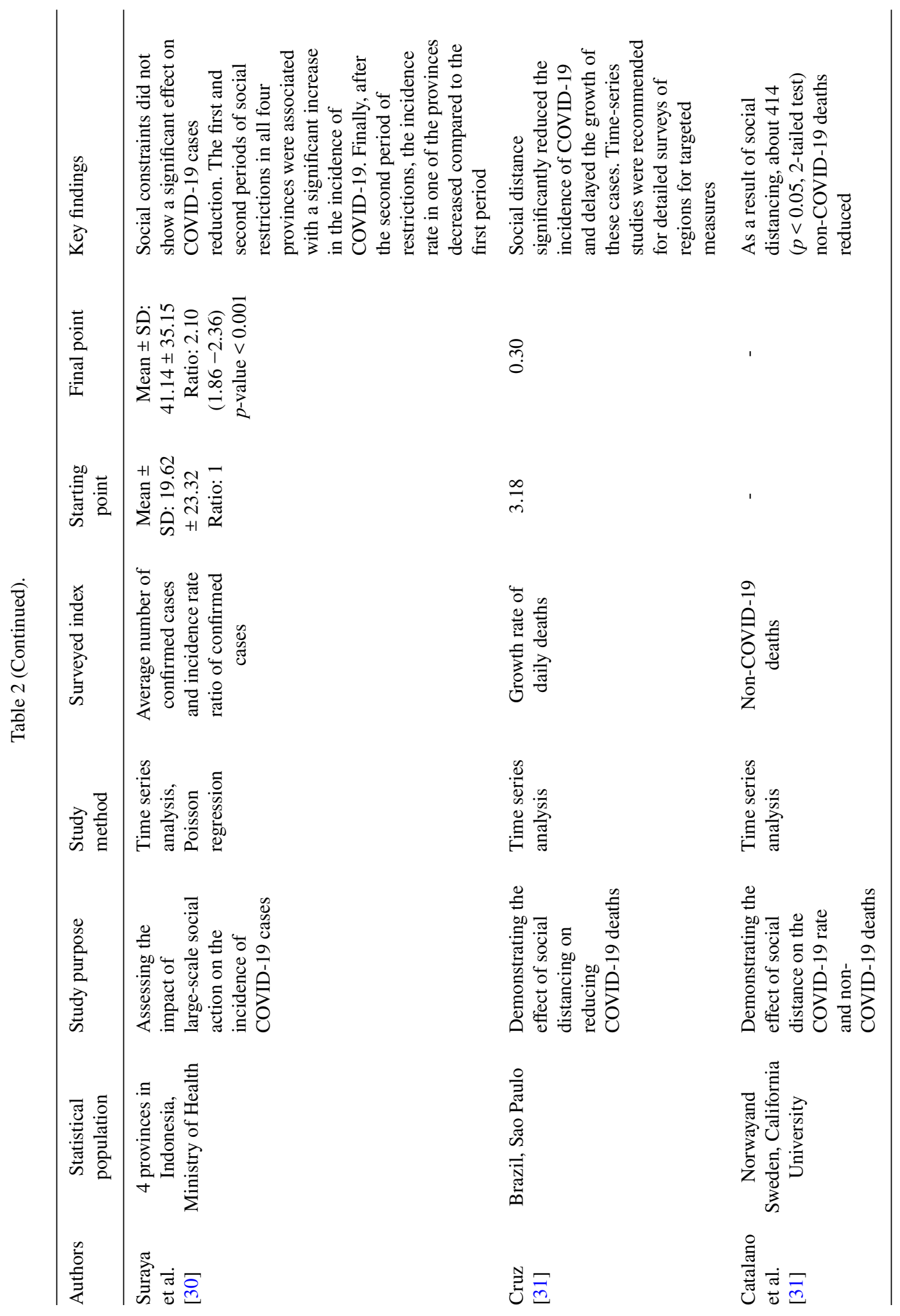




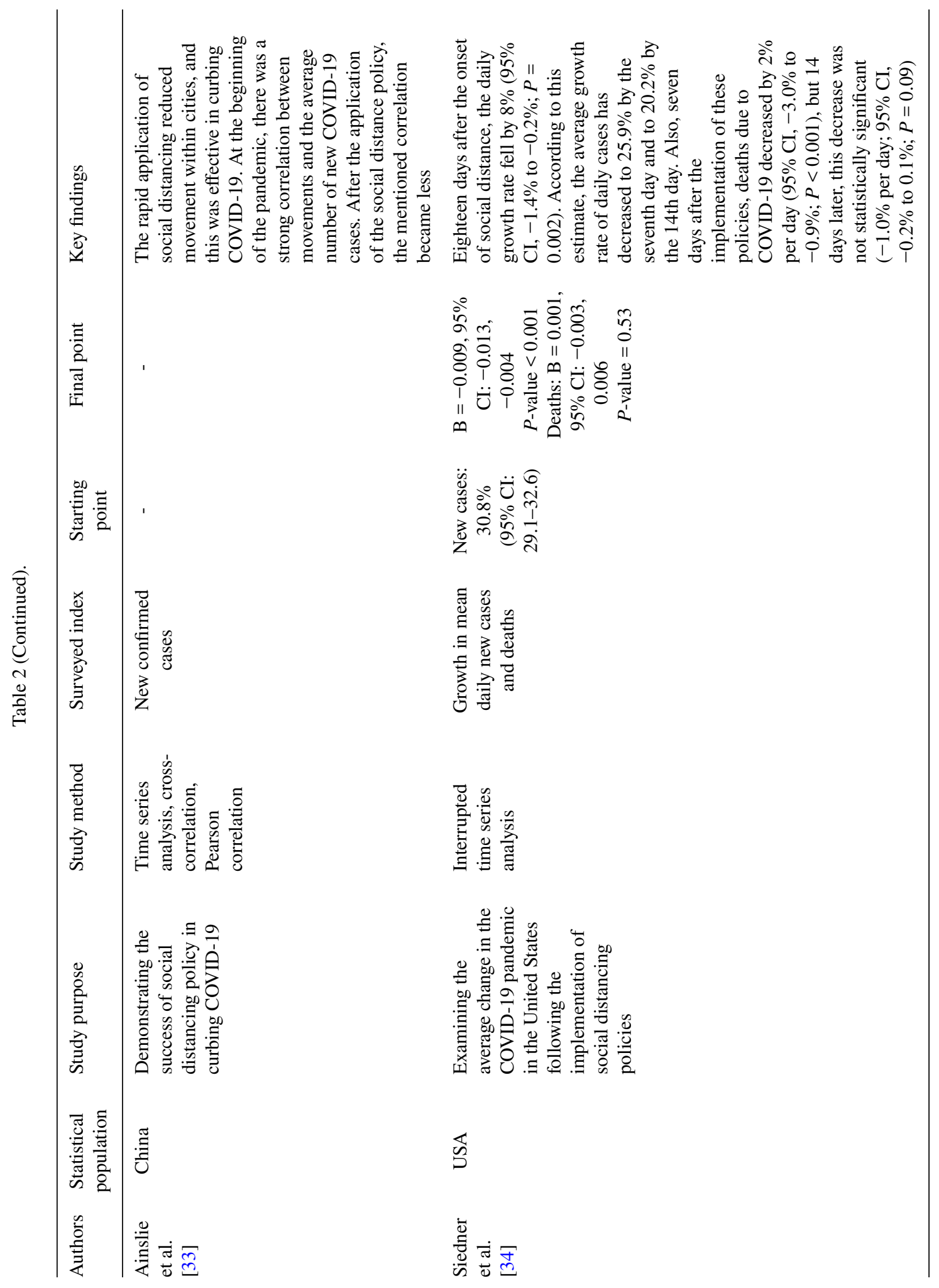




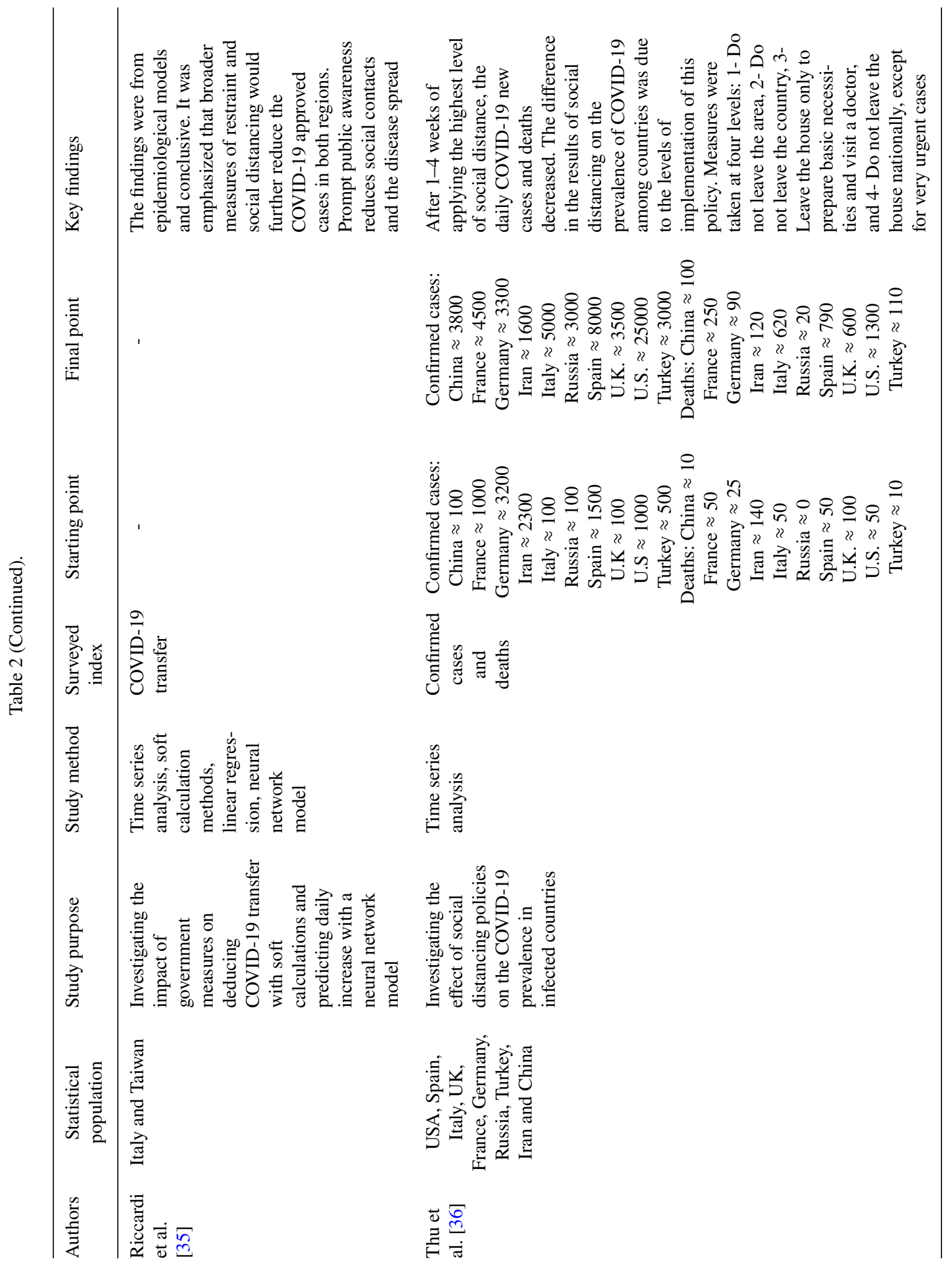


Table 3

Types of social distancing policies and strategies adopted in the studies under review

$\begin{array}{ll}\text { Restrictions } & \text { - Individual's movements restrictions } \\ & \text { - Boundary restrictions } \\ & \text { - Restrictions on the amount and method of transportation } \\ \text { - Restricting religious, cultural, and social activities } \\ \text { - Travel restrictions } \\ \text { - Cancellation and prohibition of gatherings } \\ \text { - Prohibition of cultural, sports, and religious ceremonies } \\ \text { - Prohibition of attending parks } \\ \text { - Prohibition of attending sports clubs } \\ \text { - Prohibition of attending shrines } \\ \text { - Unnecessary job groups closure } \\ \text { - Public transport closure } \\ \text { - School closure } \\ \text { - Universities closure } \\ \text { - Stadiums, cinemas and theaters closure } \\ \text { - Designing mobile apps to warn of contact with positive cases } \\ \text { - Creating campaigns to encouraging people to reduce the social activity hours } \\ \text { - Encourage families to stay home } \\ \text { - Making teleworking possible for many jobs } \\ \text { - More usage of social networks } \\ \text { - Creating the culture of following social distance by celebrities } \\ \text { - Economic support, including loans and subsidies for the closure of self-employment } \\ \text { - Considering fines for violators of the principles of social distancing } \\ \text { - Police and military oversight of the proper implementation of social distancing protocols }\end{array}$

regard is to restrict crowded and unnecessary places. In Italy and Germany, social restrictions have reduced the COVID-19 incidence and mortality [43,44]. In contrast, some of these policies were not effective enough in Indonesia, Japan, and the United States [30,45,46], which was due to a lack of attention to complementary policies of social distancing. These policies, while not completely disrupting activities, can reduce the transmission of the virus by reducing the exposure of individuals. According to the experiences of countries such as Singapore [47] and South Korea [48] in this type of low-intensity restrictions, it is especially important to accompany other protective measures, including the use of masks.

Incentive policies include economic assistance to the people of society. One of the crucial reasons that lead people not to adhere to protocols is the economic concerns especially for self-employed businesses [49]. A systematic review by Faruque Ahmed et al. Showed that social distance policies related to the workplace reduced the prevalence of influenza by an average of $23 \%$. It also reduces and delays virus attack peaks [50]. Also, a study in Saudi Arabia identified positive awareness and attitudes as a factor in people's adherence to this policy [51]. If the path to compliance with protocols is paved by governments and policies are accepted in society, individuals' commitment to adhere to protocols will increase. Incentive policies as support for other policies are essential to attracting people to participate. Providing livelihoods, communication infrastructure, informing people about the correct implementation of policies, and ensuring the effectiveness of social distance through campaigns and media are the 
Table 4

Suggestions for the effective implementation of social distance

- Early implementation of social distancing to prevent widespread outbreaks and irreversible complications

Because of the Coronavirus's high spread and transmission speed, the relatively long incubation period, many asymptomatic carriers, no universal cure; Preventive measures must be placed at the top of decisions and priorities. On the other hand, taking preventive measures early directly leads to early crisis resolution and faster recovery.

- Attracting the participation and cooperation of individuals in order to observe the social distance

Lack of people's support for the policies adopted is the main reason for their failure to implement. In addition to developing accurate and transparent guidelines and explaining them among the community, it is also necessary to attract their support and participation. Convincing people about the effectiveness of guidelines and the risks of violating those leads to a realistic attitude towards policies, which in turn leads to compliance with protocols and achieving the expected results.

- Providing financial support for vulnerable businesses

Family and business financial problems drive people to their workplaces. Being at work also increases contact between people and increases the prevalence of the virus. To prevent this, economic support for households and businesses is recommended. This support can be in the form of loans and subsidies. Such support reduces the spread of the virus in the short term and also prevents long-term damage to household and business economies.

- Paying attention to the mental health of people who stay at home

During the COVID -19 pandemic, everyone struggled with fear. In addition to these stresses, staying at home also has significant psychological effects on people. In order to prevent psychological harm to people and also to prevent people from leaving their homes uncontrollably, mental health programs for different population groups should be on the agenda of governments. These programs can include online and telephone counseling services and designing mental health software.

- Providing the needs of the elderly who live alone

Because aged people often have underlying diseases, they are more vulnerable to COVID-19. Therefore, contact with this age group should be completely cut off or control. On the other hand, the needs of the aged people may drive them out of the home, so meeting the needs of this group in the form of scheduled programs should be a priority. Buying and delivering aged people's necessities can be done by volunteer groups or government employees.

- Use of electronic communication platform to advance educational and professional goals

Nowadays, the Internet, virtual networks and online software have a significant share in all areas. Therefore, using this efficient platform can minimize face-to-face contact and at the same time promote activities. Although virtual communication is not a perfect alternative to human interaction, it is the best tool for minimizing disruption in functions. This privilege can be used in promoting career, educational, social, cultural, political and athletic activities and goals.

- Taking complementary measures such as using a face mask

Given that the effectiveness of social distance depends on the amount of virus and the duration of exposure to the virus, it is suggested that this policy be accompanied by other measures, including wearing a mask. Any measures are taken to prevent COVID-19 infection causes people to take one step back from the disease.

minimum support measures that should be implemented. Aligning other government policies such as financial, educational, informational and job policies ith social distancing policies is necessary to avoid confusion and put pressure on the people.

In relation to punitive policies, Saez et al. point to the imposition of fines on offenders [27]. The involvement of non-governmental organizations, and the use of military oversight are also summaries of policies adopted in 149 European countries to stabilize social distance [26]. Prompt implementation 
of social distancing policies is essential to curb virus transmission as soon as possible. Also, due to the emergence of such policies, it is necessary to establish legal levers to ensure the adherence of the majority until the various aspects of these policies are fully explained to the public. Also, the fact that non-adherence to social distancing policies threatens both the health and life of the individual and society, confirms the need to implement coercive policies.

Ultimately, the correct implementation of each of these policies will lead to the proper advancement of social distancing. Achieving this will reduce the burden of disease, reduce the pressure on health facilities and human resources, and as a result, hospitalized patients will enjoy better and more efficient services [29]. On the other hand, due to the importance of time in controlling the virus spread and the fact that the widespread implementation of laws and policies is time-consuming, it is necessary to protect society by implementing classified policies from the cities to the national level [52]. According to the study by Ainslie et al., China was able to quickly implement restrictions-reduction strategies, which led to economic recovery [33]. Therefore, in order to mitigate as much as possible, the psychological, environmental, and economic harms of the above policies, it is necessary to respond quickly and early. This way, the disadvantages can be reduced and the benefits of these policies can be exploited more quickly.

On the other hand, some personal and urban characteristics suchlike demographic characteristics, economic and social status, racial and ethnic composition, population density, size, geographical distance, and health infrastructure affect the COVID-19 prevalence and mortality [53-55]. Therefore, preventing the unbridled prevalence and reducing the public vulnerability requires the vigilance of policymakers in the formulation and implementation of appropriate measures for each city and region. Also, developing policies with varying degrees of intensity and levels should be on the agenda.

Social distancing as a strategy to curb the COVID-19 pandemic should be considered by policymakers and decision-makers. There are currently various programs in many countries for early detection and control of the disease. Meanwhile, social distancing can be one of the surest ways to reduce the incidence and mortality of COVID-19. Therefore, the continuation of social distancing measures is necessary to reduce and prevent further COVID-19 peaks. Various vaccines have already been approved by the WHO, but vaccine resources are limited and it is time-consuming to vaccinate in a phased and prioritized manner. At the same time, new and diverse coronavirus mutations are spreading rapidly in different countries. Therefore, social distancing and implementation of physical restraints, especially in crowded and densely populated environments, should be done extensively and seriously until the end of vaccination worldwide or the eradication of COVID-19.

The effectiveness of all social distancing policies depend on their early implementation and based on a proper analysis of the situation because late action causes a wider virus prevalence and the social involvement with complex complications.

This research is not without limitations. First, due to the heterogeneity of the data reported in the studies, it was not possible to meta-analyze the results. Second, the studies examining quarantine and lockdown were not reviewed due to the absolute prohibition of all activities.

\section{Conclusion}

Since social distancing is an inclusive strategy with different levels. This study recommends the policymakers to use different levels and intensities of relevant policies according to the characteristics of each region. Therefore, a balance is created between the characteristics of different occupational, social and age groups, and the intensity and type of policies. This way, community adherence increases and, 
consequently, the effectiveness of the policy improves. On the other hand, in order to get out of the crisis as soon as possible, the early adoption of this type of preventive policy is of great importance in maintaining public health and society's sustainability.

\section{Conflict of interest}

There are no conflicts of interest.

\section{References}

[1] Altayb HN et al. The current situation of COVID-19 in Sudan. New Microbes and New Infections. 2020;37:100746.

[2] Chang C-M et al. COVID-19: Taiwan's epidemiological characteristics and public and hospital responses. PeerJ. 2020;8:e9360.

[3] Aldila D et al. A mathematical study on the spread of COVID-19 considering social distancing and rapid assessment: The case of Jakarta, Indonesia. Chaos, Solitons \& Fractals. 2020;139:110042.

[4] Jarvis CI et al. Quantifying the impact of physical distance measures on the transmission of COVID-19 in the UK. BMC Medicine. 2020;18:1-10.

[5] Ahmed I, Ahmad M, Jeon G. Social distance monitoring framework using deep learning architecture to control infection transmission of COVID-19 pandemic. Sustainable Cities and Society. 2021;102777.

[6] Su J et al. A novel social distancing analysis in urban public space: A new online spatio-temporal trajectory approach. Sustainable Cities and Society. 2021;68:102765.

[7] W.H. Organization. Available from: https://covid19.who.int/?gclid=Cj0KCQiAmL-ABhDFARIsAKywVacaCKgBn3tR3 WBb3-tiUqV0-AAL4k6nVWcySb8h2rp-wTK2V3WGnV0aAhy2EALw_wcB. 2020.

[8] Gebru AA et al. Global public health significances, health care perception of community, treatments, prevention and control methods of Coronavirus Disease 2019 Outbreak. Human Antibodies. 2020;1-9 (Preprint).

[9] Bchetnia $\mathrm{M}$ et al. The outbreak of the novel severe acute respiratory syndrome coronavirus 2 (SARS-CoV-2): A review of the current global status. Journal of Infection and Public Health. 2020).

[10] Mahase E. Covid-19: What new variants are emerging and how are they being investigated? British Medical Journal Publishing Group. 2021).

[11] Jang WM, Jang DH, Lee JY. Social distancing and transmission-reducing practices during the 2019 coronavirus disease and 2015 Middle East respiratory syndrome coronavirus outbreaks in Korea. Journal of Korean Medical Science. 2020;35(23).

[12] W.H. Organization. Fair allocation mechanism for COVID-19 vaccines through the COVAX Facility, Final working version-9 September, 2020

[13] W.H. Organization. Transmission of SARS-CoV-2: Implications for Infection Prevention Precautions: Scientific Brief. World Health Organization; 09 July 2020.

[14] S.C.H. of the Republic. Rules and guidelines for distancing in daily life to control coronavirus disease 2019 in Korea: 3 rd version, announced on July 3, 2020. Journal of Educational Evaluation for Health Professions. 2020;17.

[15] Chong KC et al. Monitoring disease transmissibility of 2019 novel coronavirus disease in Zhejiang, China. International Journal of Infectious Diseases. 2020;96:128-30.

[16] Gupta S et al. Tracking Public and Private Responses to the COVID-19 Epidemic: Evidence from State and Local Government Actions. National Bureau of Economic Research; 2020.

[17] Kim E-A. Social distancing and public health guidelines at workplaces in Korea: Responses to coronavirus disease- 19. Safety and Health at Work. 2020;11(3):275-83.

[18] Lasry A et al. Timing of community mitigation and changes in reported COVID-19 and community mobility-four US metropolitan areas, February 26-April 1, 2020. Morbidity and Mortality Weekly Report. 2020;69(15):451-7.

[19] Liberati A et al. The PRISMA statement for reporting systematic reviews and meta-analyses of studies that evaluate health care interventions: Explanation and elaboration. Journal of Clinical Epidemiology. 2009;62(10):e1-34.

[20] Velicer WF, Fava JL. Time series analysis. Research Methods in Psychology. 2003;2.

[21] Von Elm E et al. The Strengthening the Reporting of Observational Studies in Epidemiology (STROBE) statement: Guidelines for reporting observational studies. Annals of Internal Medicine. 2007;147(8):573-7.

[22] Vokó Z, Pitter JG. The effect of social distance measures on COVID-19 epidemics in Europe: An interrupted time series analysis. GeroScience. 2020;42(4):1075-82. 
[23] Alimohamadi Y et al. Effect of social distancing on COVID-19 incidence and mortality in Iran since February 20 to May 13, 2020: An interrupted time series analysis. Risk Management and Healthcare Policy. 2020;13:1695.

[24] Varghese $\mathrm{C}, \mathrm{Xu} \mathrm{W}$. Quantifying what could have been-the impact of the Australian and New Zealand governments' response to COVID-19. Infection, Disease \& Health. 2020;25(4):242-4.

[25] de Araujo OR et al. The impact of the novel coronavirus on Brazilian PICUs. Pediatric Critical Care Medicine. 2020;21(12):1059.

[26] Islam N et al. Physical distancing interventions and incidence of coronavirus disease 2019: Natural experiment in 149 countries. BMJ. 2020;370.

[27] Saez M et al. Effectiveness of the measures to flatten the epidemic curve of COVID-19. The case of Spain. Science of the Total Environment. 2020;727:138761.

[28] Saki M et al. Interrupted time series analysis of the implementation of social distancing policy, its lifting and the mandate of wearing face masks in Iran to mitigate against COVID-19. 2020.

[29] Ghanbari MK et al. The impact of the social distancing policy on COVID-19 new cases in Iran: Insights from an interrupted time series analysis. 2020

[30] Suraya I et al. The impact of large-scale social restrictions on the incidence of covid-19: A case study of four provinces in Indonesia. Kesmas: Jurnal Kesehatan Masyarakat Nasional (National Public Health Journal). 2020).

[31] Cruz CHdB. Social distancing in São Paulo State: Demonstrating the reduction in cases using time series analysis of deaths due to COVID-19. Revista Brasileira de Epidemiologia. 2020;23:e200056.

[32] Catalano R et al. Non-COVID-19 deaths after social distancing in Norway. European Journal of Epidemiology. 2020;35(11):1021-4.

[33] Ainslie KE et al. Evidence of initial success for China exiting COVID-19 social distancing policy after achieving containment. Wellcome Open Research. 2020;5.

[34] Siedner MJ et al. Social distancing to slow the US COVID-19 epidemic: An interrupted time-series analysis. MedRxiv, 2020.

[35] Riccardi A et al. Optimisation of non-pharmaceutical measures in COVID-19 growth via neural networks. IEEE Transactions on Emerging Topics in Computational Intelligence. 2021;5(1):79-91.

[36] Thu TPB, Ngoc PNH, Hai NM. Effect of the social distancing measures on the spread of COVID-19 in 10 highly infected countries. Science of the Total Environment. 2020;742:140430.

[37] Kuniya T, Inaba H. Possible effects of mixed prevention strategy for COVID-19 epidemic: Massive testing, quarantine and social distancing. AIMS Public Health. 2020;7(3):490.

[38] Dao TL, Nguyen TD. Controlling the COVID-19 pandemic: Useful lessons from Vietnam. Travel Medicine and Infectious Disease. 2020;37:101822.

[39] Singhal T. A review of coronavirus disease-2019 (COVID-19). The Indian Journal of Pediatrics. 2020;87(4):281-6.

[40] Morley CP et al. Social distancing metrics and estimates of sars-cov-2 transmission rates: Associations between mobile telephone data tracking and r. Journal of Public Health Management and Practice. 2020;26(6):606-12.

[41] Angoulvant $\mathrm{F}$ et al. Coronavirus disease 2019 pandemic: Impact caused by school closure and national lockdown on pediatric visits and admissions for viral and nonviral infections-a time series analysis. Clinical Infectious Diseases. 2021;72(2):319-22.

[42] Auger KA et al. Association between statewide school closure and COVID-19 incidence and mortality in the US. JAMA. 2020;324(9):859-70.

[43] Chintalapudi N, Battineni G, Amenta F. COVID-19 virus outbreak forecasting of registered and recovered cases after sixty day lockdown in Italy: A data driven model approach. Journal of Microbiology, Immunology and Infection. 2020;53(3):396-403.

[44] Bönisch S et al. Effects of coronavirus disease (COVID-19) related contact restrictions in Germany, March to May 2020, on the mobility and relation to infection patterns. Frontiers in Public Health. 2020;8:619.

[45] Iwata K, Doi A, Miyakoshi C. Was school closure effective in mitigating coronavirus disease 2019 (COVID-19)? Time series analysis using Bayesian inference. International Journal of Infectious Diseases. 2020;99:57-61.

[46] Al-Hasan A, Khuntia J, Yim D. Threat, coping, and social distance adherence during COVID-19: Cross-continental comparison using an online cross-sectional survey. Journal of medical Internet Research. 2020;22(11):e23019.

[47] Wang J et al. Mask use during COVID-19: A risk adjusted strategy. Environmental Pollution. 2020;115099.

[48] Lim S et al. Face masks and containment of COVID-19: Experience from South Korea. Journal of Hospital Infection. 2020;106(1):206-7.

[49] Hatchett RJ, Mecher CE, Lipsitch M. Public health interventions and epidemic intensity during the 1918 influenza pandemic. Proceedings of the National Academy of Sciences. 2007;104(18):7582-7. 
[50] Ahmed F, Zviedrite N, Uzicanin A. Effectiveness of workplace social distancing measures in reducing influenza transmission: A systematic review. BMC Public Health. 2018;18(1):1-13.

[51] Alotaibi $\mathrm{N}$ et al. The extent of commitment of Saudis during holy Ramadan to social distancing measures required for the prevention of transmission of COVID-19. Journal of Community Health. 2020;1-10.

[52] Mollalo A, Rivera KM, Vahabi N. Spatial statistical analysis of pre-existing mortalities of 20 diseases with COVID-19 mortalities in the continental United States. Sustainable Cities and Society. 2021;67:102738.

[53] Maiti A et al. Exploring spatiotemporal effects of the driving factors on COVID-19 incidences in the contiguous United States. Sustainable Cities and Society. 2021;68:102784.

[54] $\mathrm{Li} \mathrm{B}$ et al. Built environment and early infection of COVID-19 in urban districts: A case study of Huangzhou. Sustainable Cities and Society. 2021;66:102685.

[55] Yang T et al. What determines city's resilience against epidemic outbreak: Evidence from China's COVID-19 experience. Sustainable Cities and Society. 2021;102892. 